\title{
Percepções de Estudantes Universitários sobre a Realização de Atividades Extracurriculares na Graduação
}

\author{
Clarissa Tochetto de Oliveira \\ Universidade Federal do Rio Grande do Sul, RS, Brasil. Universidade do Vale do Rio dos Sinos, RS, Brasil. \\ Ana Cristina Garcia Dias \\ Universidade Federal de Santa Maria, RS, Brasil.
}

\begin{abstract}
Resumo: O objetivo deste estudo foi identificar o que induz os estudantes universitários a buscarem a realização de atividades extracurriculares, em que tipo de atividades se envolvem e como avaliam esse envolvimento. Participaram desta pesquisa 24 estudantes de dois cursos de graduação (Psicologia e Economia) de uma universidade pública do sul do Brasil. As informações foram coletadas por meio de uma entrevista semiestruturada individual e foram submetidas à análise de conteúdo temática. As informações sugerem que os estudantes percebem a necessidade de buscar conhecimentos e oportunidades para complementar sua formação. Para tanto, envolvem-se em trabalho voluntário, projetos de pesquisa e extensão, e estágios. De maneira geral, as atividades extracurriculares são avaliadas como positivas, pois possibilitam a aplicação dos conteúdos estudados em sala de aula. Contudo, os estudantes encontram dificuldades na procura por atividades não obrigatórias. Conclui-se que a exploração profissional pode auxiliar no envolvimento dos estudantes com o curso, além de ajudar no amadurecimento de comportamentos autodidatas, que são benéficos especialmente para $o$ período pós-formatura, no qual há a preocupação de se inserir no mercado de trabalho.

Palavras-chave: Estudantes Universitários, Ensino Superior, Atividade Extracurricular.
\end{abstract}

\section{College Students' Perceptions Regarding Involvement in Extra-curricular Activities During College}

\begin{abstract}
The aim of this study was to identify what makes college students take part in extracurricular activities, what kind of activities they join, and how they evaluate this experience. The participants were 24 students of Psychology and Economy from a university of Southern Brazil. The information was gathered through private semi structured interviews and was submitted to content analysis. Results showed that students of the sample identify the need to seek knowledge and opportunities to complement their educational background. To do so, they take part in volunteer work, research and extension projects, and internships. In general, participants evaluated extracurricular activities positively, once they make it possible to apply knowledge built in classroom into practice. However, the students face difficulties to join extracurricular activities. We conclude that professional exploration may contribute to students' engagement with the course as well as to the development of self-taught behaviors, which are useful especially after graduation when students are concerned about joining the labor market. Keywords: College Students, Higher Education, Extracurricular Activity,
\end{abstract}




\title{
Percepciones de Estudiantes Universitarios sobre Participación en Actividades Extracurriculares en la Pregrado
}

\begin{abstract}
Resumen: El objetivo de este estudio fue identificar lo que induce a los estudiantes universitarios a realizar actividades extracurriculares, en qué tipo de actividades están involucrados y cómo evalúan su participación. Participaron de esta investigación 24 estudiantes de dos carreras (Psicología y Economía) de una universidad del sur de Brasil. Las informaciones fueron recolectadas por medio de una entrevista semiestructurada individual y fueron sometidas a análisis de contenido temático. Los alumnos percibieron la necesidad de buscar conocimientos y oportunidades para complementar su formación. Con este fin, se involucran en trabajo voluntario, proyectos de investigación y extensión, y pasantías. En términos generales, las actividades extracurriculares son evaluadas como positivas, ya que muestran la aplicación de los contenidos estudiados en clase. Sin embargo, los universitarios encuentran dificultades en la búsqueda para encontrar actividades no obligatorias. Se concluye que la exploración profesional puede auxiliar en el involucramiento de los académicos con la carrera, además de ayudar en la maduración de comportamientos autodidactas, que son beneficiosos principalmente para el período después del pregrado, en el que hay la preocupación de insertarse en el mercado laboral.
\end{abstract}

Palabras clave: Estudiantes Universitarios, Educación Superior, Actividad Extracurricular.

\section{Introdução}

A transição do ensino médio para o ensino superior, assim como outras transições educacionais (por exemplo, a transição do ensino fundamental para o ensino médio), traz consigo modificações nos métodos de ensino, nos vínculos estabelecidos, nos papéis sociais e na rotina dos estudantes (Alves, Gonçalves, \& Almeida, 2012). Essas alterações exigem flexibilidade e autonomia dos estudantes para que eles se ajustem às novas responsabilidades desse novo contexto (Alves et al., 2012, Soares, Almeida, \& Ferreira, 2002).

Ser autônomo significa que o indivíduo apresenta um sentido claro, seguro e estável de si mesmo, manifestando comportamentos coordenados e intencionais na busca de objetivos pessoais e/ou sociais. O processo de autonomização envolve três dimensões: (1) independência emocional, traduzida na ausência da necessidade constante de segurança, afeto e aprovação por parte de figuras significativas, como, pais e amigos, (2) independência instrumental, refletida na capacidade e na confiança que o estudante tem de, por si mesmo, executar um conjunto de atividades e de ser capaz de resolver os problemas que daí podem decorrer sem a ajuda sistemática dos outros, e (3) interdependência, expressa pelo reconhecimento e pela aceitação de que não se pode rece- ber benefícios de uma estrutura social sem contribuir para ela. À medida que ocorre o desenvolvimento de cada uma dessas dimensões, os indivíduos manifestam um estilo de vida mais independente, seguro e ativo. Eles se tornam cada vez mais capazes de definir e buscar objetivos pessoais e/ou sociais e de se relacionar consigo, com os outros e com o mundo de uma forma mais satisfatória (Soares et al., 2002).

Entende-se que um maior nível de autonomia associa-se a uma adaptação acadêmica mais adequada (Soares et al., 2002). A adaptação acadêmica refere-se ao quão bem o universitário consegue corresponder às demandas acadêmicas impostas a ele e, a partir disso, obter êxito no Ensino Superior (Credé, \& Niehorster, 2012). O modo como os estudantes se ajustam à vida universitária é determinante para a vivência de melhores ou piores experiências relacionadas à sua formação profissional e ao desenvolvimento psicossocial nesse período (Santos, Mognon, Lima, \& Cunha, 2011, Soares et al., 2014; Teixeira, Dias, Wottrich, \& Oliveira, 2008). A autonomia permite que o estudante se conscientize sobre a necessidade de busca constante de conhecimento e de construção de sua identidade social e profissional. Cabe lembrar que nem sempre a grade curricular dos cursos de graduação oferece para os alunos todas as 
habilidades necessárias para a atuação profissional, sendo necessária a busca de habilidades e experiências complementares. Um maior senso de autonomia permite que o estudante busque sanar deficiências com as quais se depara em sua formação através da busca e engajamento em atividades extracurriculares (Bridi, 2010).

De fato, o envolvimento do estudante universitário em atividades extracurriculares vem ganhando destaque em pesquisas brasileiras (Bardagi, \& Hutz, 2012; Fior, \& Mercuri, 2009; Peres, Andrade, \& Garcia, 2007; Santos et al., 2011) e internacionais (Baker, 2008; Hu \&Wolniak, 2010; Huang, \& Chang, 2004; Stevenson, \& Clegg, 2011; Tavares, 2012) como um fator relevante a ser considerado no processo de adaptação ao contexto universitário. As atividades extracurriculares, também denominadas complementares ou não obrigatórias, incluem a participação em monitoria, iniciação científica, projetos de extensão, grupos de estudos e pesquisa, nos órgãos de representação estudantil, em congressos e eventos científicos, estágios remunerados ou não remunerados, entre outras (Bardagi, \& Hutz, 2012; Fior, \& Mercuri, 2009).

A análise dos estudos brasileiros permite afirmar que a inserção em atividades extracurriculares costuma estar associada à qualidade da identidade profissional percebida e ao comprometimento do estudante com a formação. Os estágios, por exemplo, são vistos pela maioria dos estudantes como uma possibilidade de preparação para o exercício do papel de trabalhador e como uma forma de ajudar no desenvolvimento do pensamento criativo, das habilidades sociais e do estabelecimento de contatos interpessoais (Bardagi, \& Hutz, 2012).

Já as atividades desenvolvidas no ambiente universitário, a saber, participação em projetos de pesquisa, projetos de extensão, grupos de estudos e monitoria, permitem o estabelecimento de contato mais próximo com colegas e professores. Além disso, oportuniza a exploração de aspectos da formação que muitas vezes não são contemplados pelo currículo dos cursos de graduação, como o entendimento do processo de pesquisa, o qual engloba desde a sua idealização e execução até a publicação de artigos em periódicos científicos. De fato, a movimentação do estudante pelo ambiente do curso, preenchendo seus horários com atividades complementares, possibilita conhecer novas realidades e motiva os universitários em relação à rotina acadêmica (Teixeira et al., 2008).
De maneira geral, estudantes brasileiros relataram que as atividades extracurriculares proporcionam aquisição de conhecimentos e novas experiências que complementam o currículo básico, satisfazem o desejo de vivenciar a profissão escolhida, e, em alguns casos, são uma fonte de renda para o estudante (Peres et al., 2007). A participação em atividades extracurriculares pode resultar em mais satisfação e compromisso com o curso, aprimoramento das habilidades de liderança e facilidade de estabelecer relacionamentos interpessoais (Fior, \& Mercuri, 2009).

Os resultados encontrados nos estudos internacionais sobre o tema vão ao encontro dos resultados obtidos nas pesquisas brasileiras. Os estudantes que dedicam mais tempo à dimensão acadêmica de suas vidas, por meio da participação em atividades extracurriculares, apresentam mais qualidade e sucesso nas vivências universitárias bem como melhor rendimento acadêmico (Baker, 2008; Tavares, 2012). Além disso, exibem melhores competências interpessoais e de estudo, bem-estar físico e psicológico, mais satisfação com o curso e com a instituição e conhecimento mais aprofundado das estruturas e dos serviços que existem na universidade (Tavares, 2012). A combinação entre atividades obrigatórias e não obrigatórias contribui para o aperfeiçoamento cognitivo e afetivo dos estudantes (Huang, \& Chang, 2004).

Estudos internacionais também enfatizam a importância do envolvimento em atividades extracurriculares para alavancar a empregabilidade dos jovens (Hu, \& Wolniak, 2010; Stevenson, \& Clegg, 2011). Isso porque a participação em atividades complementares demonstra que os jovens se preocuparam com a qualidade de sua formação e foram proativos na busca por experiências que não ficassem restritas aquelas oferecidas pela grade curricular dos cursos, o que faz com que os empregadores acreditem que eles apresentarão postura semelhante quando contratados. Ademais, a participação nesse tipo de atividade pode contribuir para o aumento salarial após a entrada no mercado de trabalho, já que é valorizada pela maioria das empresas (Hu, \&Wolniak, 2010).

A literatura especializada mostra os benefícios da participação em atividades extracurriculares, tais como menor probabilidade de evasão, mais satisfação com as experiências na universidade, maior integração ao contexto universitário e criação da identidade profissional, elementos importantes para a adaptação acadêmica do estudante (Busseri et al., 2010; Fior, \& Mercuri, 2009). 
Sabe-se que os estudantes tendem a valorizar a realização de atividades complementares, uma vez que as percebem como uma oportunidade para praticar os conhecimentos apresentados pelos professores em aula e vivenciar a profissão escolhida (Teixeira, \& Gomes, 2005). No entanto, não fica claro na literatura sobre a temática como ocorre o processo de envolvimento em atividades extracurriculares. A partir disso, o objetivo deste estudo foi identificar o que induz os estudantes universitários a buscarem a realização de atividades extracurriculares, em que tipo de atividades se envolvem e como avaliam esse envolvimento.

Supõe-se que os estudantes procuram se envolver em atividades complementares na tentativa de entender a aplicabilidade das teorias e dos conceitos estudados em aula. Acredita-se que esse envolvimento gera um sentimento de pertença à categoria profissional na qual o universitário irá se enquadrar após finalizar a graduação. Possivelmente, isso gera a identificação do estudante com o curso, uma vez que para atuar na profissão escolhida é necessário concluir a graduação. Isso pode favorecer a adaptação acadêmica dos graduandos devido ao desejo de poder exercer as atividades que tal profissão possibilita, as quais foram previamente colocadas em prática por meio do envolvimento em atividades extracurriculares.

\section{Método}

Trata-se de uma pesquisa de delineamento qualitativo exploratório (Piovesan, \& Temporini, 1995).

\section{Participantes}

Participaram deste estudo 24 estudantes (17 mulheres) de uma universidade pública localizada no interior do Rio Grande do Sul. Os estudantes eram provenientes dos cursos de Psicologia (seis calouros e sete formandos) e Economia (cinco calouros e seis formandos). As idades estavam no intervalo entre 17 e 26 anos. Os nomes dos participantes foram substituídos por nomes fictícios a fim de preservar seu anonimato.

\section{Procedimentos e instrumentos}

Após a pesquisa ser aprovada pelo comitê de ética da universidade na qual o estudo foi realizado (CAAE $\mathrm{n}^{\circ}$ 0126.0.243.000-08), seus objetivos foram apresentados aos coordenadores e professores dos cursos de Psicologia e Economia. A mesma explicação foi oferecida aos alunos, convidando-os a participar do estudo, no contato realizado em sala de aula. Não houve desistência por parte dos alunos que se propuseram a colaborar com a pesquisa. $\mathrm{O}$ número de participantes foi definido pelo critério de saturação das informações, ou seja, a coleta dos dados foi interrompida quando as informações se tornaram repetitivas (Minayo, 1992).

Uma aluna de graduação da Psicologia treinada realizou entrevistas semiestruturadas individuais, que foram realizadas nas dependências da universidade em uma sala reservada, garantindo o sigilo das informações fornecidas e a privacidade. Durante a entrevista, só estavam presentes a entrevistadora e o/a participante. Foi realizada apenas uma entrevista por participante. Os estudantes foram questionados sobre sua trajetória acadêmica de maneira geral e sobre os diversos aspectos inerentes à adaptação universitária. Foram utilizadas questões abertas e fechadas, tais como: como você descreveria sua trajetória na universidade? Como foi sua adaptação ao longo do curso? Quais foram as maiores dificuldades que você encontrou ao longo do curso? Como é sua relação com os colegas? Como você descreve sua relação com os professores? As informações analisadas neste trabalho são relativas às perguntas feitas sobre a busca de conhecimentos e sobre o envolvimento em atividades extracurriculares: Você precisou buscar aprendizados por conta própria (fora da sala de aula)? Que atividades foram buscadas? Como você percebe essa busca por outras atividades que complementem a sua formação? Essas perguntas foram realizadas depois das perguntas apresentadas anteriormente.

\section{Análise das informações}

As entrevistas foram gravadas e, posteriormente, transcritas na íntegra. As informações foram analisadas por uma aluna de pós-graduação em Psicologia com experiência prévia em análise qualitativa. As respostas foram submetidas à análise de conteúdo temática (Bardin, 2011). Primeiramente as respostas de todos os participantes foram reunidas de acordo com as perguntas realizadas. Em seguida, fez-se uma leitura flutuante para familiarização com os conteúdos manifestados. Após, as informações foram reunidas em grupos conforme semelhança de seus conteúdos. Esses 
grupos foram organizados em três categorias definidas a priori com base no objetivo do estudo: (1) motivos para a busca de atividades extracurriculares, (2) tipos de atividades extracurriculares e (3) avaliação do envolvimento em atividades extracurriculares. Por fim, outra aluna de pós-graduação em Psicologia, também com experiência em análise qualitativa, revisou o resultado da análise para identificar possíveis falhas na categorização, como categorias não excludentes. Não foram encontradas ambiguidades.

\section{Resultados e discussão}

Verificou-se que a busca por atividades extracurriculares, ou seja, para além do que é proposto pelo currículo dos cursos, parece iniciar a partir do reconhecimento da necessidade de assumir maior responsabilidade pelo próprio percurso profissional. Os estudantes que percebem que nem todos os conteúdos necessários à sua formação profissional adequada podem ser trabalhados em sala de aula tendem a buscar conhecimentos de outras formas. Essa busca frequentemente ocorre por meio da participação em atividades extracurriculares e pode contribuir para maior identificação dos estudantes com o curso de graduação e, portanto, favorecer a adaptação acadêmica dos mesmos. De maneira geral, os estudantes consideram as oportunidades de enriquecer a formação como algo positivo, embora possam encontrar alguns obstáculos para conciliar a graduação e as atividades extracurriculares nas quais se envolvem. Observou-se, ainda, que pode haver diferenças para calouros e formandos nesse processo. Essas informações serão apresentadas e discutidas a seguir.

\section{Motivos para a busca de atividades extracurriculares}

Foi realizada uma pergunta fechada aos estudantes questionando se eles haviam buscado conhecimentos por conta própria. Constatou-se que a maioria dos participantes (20 informantes) deste estudo identificou a necessidade de buscar conhecimento por conta própria durante a graduação, seja no início ou no final do curso, como motivo para se envolver em atividades extracurriculares. Entretanto, alguns calouros (quatro) revelaram não ter sentido essa necessidade. A percepção dessa necessidade pode surgir no decorrer da graduação, uma vez que todos os formandos entrevistados afirmaram buscar conhecimentos em outras fontes além da sala de aula.

Sabe-se que o ingresso no ensino superior é acompanhado não só por tarefas relacionadas ao desenvolvimento, mas também por um ritmo de trabalho diferente do ensino médio, que requer maior uso das capacidades cognitivas e, principalmente, de uma atitude mais autônoma frente ao aprendizado (Soares, Poube, \& Mello, 2009). Assim, pode-se compreender por que a maioria dos participantes da presente pesquisa indicou a necessidade de assumir uma postura mais ativa na busca de conhecimentos que, muitas vezes, está associada à identificação de oportunidades de aprendizagem fora da sala de aula (Teixeira et al., 2008).

Salienta-se a diferença identificada entre calouros e formandos no que se refere à percepção da necessidade de se envolver em atividades extracurriculares. A literatura já aponta que a participação em atividades como estágios, bolsas de pesquisa e monitorias é esperada de graduandos em fase final de curso, e que aqueles que as buscam apresentam níveis mais elevados de autoeficácia (Sparta, Bardagi, \& Andrade, 2005). No entanto, não fica claro o que faz com que as pessoas durante a graduação cheguem a essa conclusão em diferentes momentos. As falas dos participantes desse estudo, quando questionados sobre a razão que os levou a buscar conhecimentos extraclasse, sugerem que os motivos são a percepção de que nem todos os conteúdos são abordados em sala de aula, da falta de domínio de alguns conteúdos e da necessidade de entrar em contato com a profissão: "Nem tudo vai ser abordado dentro da sala de aula. Se tu queres saber um pouco de alguma outra coisa, tu vai ter que buscar" (Aline, caloura, Economia); "Fez falta os conteúdos iniciais que eu não dei valor [...]. Outra coisa que me ajudou a curtir mais o curso foi que eu comecei a buscar fora. Logo no início não tinha prática" (Nádia, formanda, Psicologia).

Os participantes desse estudo parecem perceber que os conteúdos trabalhados em sala de aula não bastam para serem bons profissionais. O conhecimento básico de cada profissão pode ser adquirido por meio do currículo oferecido pelo curso de graduação. Contudo, o desenvolvimento de habilidades e a aquisição de experiências só podem ser conquistados por meio da participação em atividades extracurriculares. Ademais, estas podem contribuir para que os alunos assumam responsabilidade sobre seu futuro e 
suas decisões (Rawat, Rastogi, Jaiswal, \& Nigam, 2014). Logo, a combinação do currículo básico do curso de graduação com a realização de atividades extracurriculares pode resultar no desenvolvimento e sucesso dos alunos (Elias, \& Drea, 2013).

\section{Tipos de atividades extracurriculares}

Observou-se que os calouros e formandos entrevistados compartilham de fontes nas quais buscaram conhecimentos. Os participantes afirmaram recorrer à leitura de artigos e livros, além de procurar materiais na internet, para complementar os conteúdos abordados em aula e para realizar os trabalhos acadêmicos. Nesse sentido, os professores desempenham um papel importante ao indicar em quais materiais os alunos podem buscar as informações que julgarem necessárias.

Verificou-se que os estudantes também se envolvem com atividades que contribuem para o desenvolvimento de habilidades, como a realização de cursos de informática e de trabalhos voluntários em organizações não governamentais (ONGs), a fim de enriquecer sua formação. Por exemplo, a participação em cursos de informática ensina o aluno a utilizar programas específicos que podem ser úteis na sua área de formação. Já o trabalho voluntário em uma ONG, descrito a seguir pela entrevistada, simula um ambiente empresarial, no qual os estudantes podem aprender a trabalhar em equipe, a coordenar um grupo de pessoas e a falar em público: "O teu professor vai ser importante pra te indicar. Eu busquei pedindo bibliografia pra alguns professores, às vezes na internet e nas bibliotecas da universidade" (Lauro, calouro, Psicologia); "Eu entrei pra [ONG] e já comecei a me ocupar bastante, porque eu estou aqui pra me desenvolver. É como se fosse uma empresa, aí tem vários times, várias áreas, e tu pode trabalhar na que mais te interessa, de acordo com o teu perfil profissional" (Janaina, caloura, Psicologia); "Desde a questão de informática, que quando eu entrei só vi o básico. Tive curso, mas eu não tive computador, eu tive que correr atrás disso" (Aline, formanda, Economia).

Pode-se compreender a busca por atividades extracurriculares como um viés do comportamento exploratório que seguidamente é realizado na fase de escolha profissional, uma vez que ambos se referem a atitudes relacionadas à solução de problemas por meio do acesso a informações (Jordaan, 1963; Ogushi,
\& Bardagi, 2015). Assim, a adoção dessa prática no início do curso e sua manutenção ao longo da trajetória universitária podem ser úteis para facilitar o processo de adaptação e envolvimento acadêmico, por meio da participação em atividades que exigem responsabilidade, oportunizam contato com professores e pares e possibilitam conhecer novas realidades (Huang, \& Chang, 2004; Reaves, Hinson, \& Marchant, 2010; Teixeira et al., 2008).

Além disso, a participação em atividades extracurriculares pode subsidiar escolhas de estágios e áreas de interesse para especializações, contribuindo, assim, para a construção de planos de carreira (Bardagi, \& Boff, 2010; Teixeira, Bardagi, \& Hutz, 2007). O envolvimento com atividades complementares ainda está associado com a autoeficácia profissional, com o comprometimento (Bardagi, \& Boff, 2010) e com a satisfação dos estudantes com sua profissão. Alunos mais engajados em atividades não obrigatórias tendem a ser mais satisfeitos com a profissão escolhida do que estudantes menos envolvidos com esse tipo de oportunidade. Essa relação pode ser explicada pela proximidade e conhecimento sobre as opções de atuação na carreira escolhida, mais comum entre os estudantes que participam de atividades extracurriculares (Bardagi, \& Hutz, 2010).

Identificou-se que os formandos entrevistados relataram um maior número de opções nas quais buscavam aprendizagem complementar quando comparados aos calouros. Os estudantes que estavam no final da graduação mencionaram participar de eventos (palestras, oficinas, congressos), projetos de pesquisa e extensão e, ainda, de estágios extracurriculares. Essa diferença entre calouros e formandos pode ser justificada pelo tempo de curso e de exploração de oportunidades, ou seja, o repertório maior de opções de atividades complementares de alunos concluintes pode estar relacionado ao maior tempo em que estes estudantes estão frequentando o curso universitário e explorando as oportunidades extracurriculares, pelo menos três anos a mais do que os alunos que estão no início do curso: "Eu fiz um ciclo de oficinas de formação de saúde pública. O resto é palestras..." (Hélio, formando, Psicologia); "Eu já fui bolsista de um projeto de pesquisa, então isso me fez buscar, ir bem além do que eu via na aula, buscar livros, material na internet. Fora algum trabalho que a gente tenha que pesquisar alguma coisa a mais" (Camila, formanda, Economia); "A primeira atividade que eu busquei 
fora era um projeto de extensão. Depois eu busquei o laboratório de pesquisa, fiquei dois anos trabalhando" (Helena, formanda, Psicologia); "Logo no início assim não tinha prática, então eu fiz estágio extra, procurei empresa fora daqui" (Nádia, formanda, Psicologia).

De fato, formandos tendem a apresentar níveis mais elevados de comportamentos exploratórios quando comparados a calouros (Igue, Bariani, \& Milanesi, 2008; Oliveira, \& Coleta, 2008; Sparta et al., 2005; Teixeira et al., 2007). Isso pode ser explicado não só pela existência de atividades que favorecem essa exploração, como as presentes nos últimos anos do curso (estágio, monografia), mas também pela preocupação dos estudantes em escolher uma área de atuação para a inserção no mercado de trabalho (Igue et al., 2008; Oliveira, \& Coleta, 2008; Teixeira et al., 2007) e pela exigência de aperfeiçoamento de habilidades e competências (Bardagi, \& Hutz, 2010). Dessa forma, espera-se que os estudantes de final de curso se envolvam em estágios extracurriculares, em projetos de pesquisa e em atividades de monitorias ou extensão (Sparta et al., 2005). Tanto é verdade que estágios extracurriculares e projetos de pesquisa são indicados como as atividades mais frequentadas pelos estudantes em contexto nacional e internacional (Bardagi, \& Boff, 2010; Thiry, Laursen, \& Hunter, 2011).

\section{Avaliação das atividades extracurriculares}

A percepção dos participantes deste estudo sobre seu envolvimento em atividades extracurriculares pode variar, embora reconheçam a importância dessas atividades. Enquanto alguns estudantes apontam a participação como algo necessário e, até mesmo, positivo, outros alunos identificam dificuldades associadas a este processo.

Os calouros e formandos entrevistados avaliaram a participação em atividades extracurriculares como necessária, uma vez que o curso não pode abordar com profundidade todos os conteúdos pertinentes à profissão. Ademais, os alunos perceberam que a iniciativa de buscar conhecimentos pode ser um diferencial para os profissionais continuarem atualizados após a formatura, quando consideram que cada um assumirá responsabilidade total pelo desenvolvimento da própria carreira: "Nem tudo vai estar na sala de aula, se você quer ser um bom profissional, vai ter que buscar" (Renata, caloura, Economia); "Acho normal, depois de se formar, tu tem que estar sempre buscando novos conhecimentos, se não vai ficando ultrapassado, a profissão não para de evoluir" (Paula, caloura, Psicologia); "É impossível o curso oferecer tudo que um aluno precisa" (Carolina, formanda, Economia).

Os estudantes parecem reconhecer a oferta de uma formação básica e generalista como principal função do curso de graduação. A busca por conhecimentos específicos nas áreas de interesse é percebida como uma responsabilidade pessoal. Fica evidente que a participação em atividades extracurriculares contribui para complementar o aprendizado ofertado em sala de aula (Thiry et al., 2011). Além disso, os estudantes percebem a necessidade de aprender a buscar conhecimento de forma mais autônoma, já que serão responsáveis pela sua atualização após a conclusão da graduação. Assim, o estudo começa a ser compreendido como uma aquisição de conhecimentos que serão utilizados na vida (Teixeira et al., 2008).

Os participantes deste estudo ainda atribuíram diversas vantagens à busca por atividades que podem contribuir para seu aprendizado. Os estudantes relataram que a participação em atividades não obrigatórias (projetos, estágios, eventos) contribui para a construção de sua autonomia profissional, uma vez que podem aprofundar os conhecimentos dos conteúdos que consideram interessantes e importantes para a sua formação. O envolvimento com essas oportunidades ainda pode auxiliá-los na exploração das possíveis áreas de atuação na profissão escolhida, no desenvolvimento de habilidades e na preparação para o trabalho de conclusão de curso.

"Dessas experiências fora, ajuda a pensar melhor, porque isso não vem só do conhecimento teórico, da sala de aula, essa é uma formação que não é o livro que vai te dar. [...] A gente trabalhava a questão da denúncia da violência com criança e adolescente, é uma coisa que a gente não vê no curso" (Letícia, formanda, Psicologia).

"Uma das vantagens das atividades extracurriculares é que elas nos dão possibilidade de nos identificarmos com outras coisas" (Carlos, formando, Psicologia).

"Eu descobri que é uma área que eu gosto, tanto que eu pretendo fazer residência depois” (Hélio, formando, Psicologia). 
"Considero fundamental, aprendi muito buscando fora. [...] Tu aprende a lidar com pessoas, tu aprende a falar, a ficar quieto, a ouvir, a cumprir horários, a cumprir metas. Em qualquer área que seja, tu aprende um monte, tu consegues associar bastante a teoria, mas quando tu vai colocar na prática muda bastante" (Carla, formanda, Psicologia).

"É bom tu não depender só do professor, tu ter as tuas fontes e procurar saber mais é sempre interessante. Apesar de ser muito bom que a gente procure, até pela monografia mesmo, que a gente tem que fazer no final do curso, não vai ser a tua orientadora que vai dizer o que tu tem que fazer" (Fabiana, formanda, Economia).

Outra vantagem citada pelos participantes corresponde à possibilidade de compreender a aplicação prática das teorias estudadas em aula. É possível que essa oportunidade possua uma importância ainda maior nos semestres iniciais da graduação, período que, segundo os entrevistados, pode despertar o desejo de evasão em função do maior distanciamento da profissão. Nesse contexto, o envolvimento em atividades complementares (como projetos de pesquisa e extensão, de estágios extracurriculares e de eventos) permite maior compreensão dos conteúdos estudados e maior contato com a profissão, o que pode contribuir para a permanência dos alunos iniciantes no curso.

"Acho que contribuem bastante, porque são exemplos depois que tu pode trazer dentro do conteúdo que tu estás estudando" (Bruna, caloura, Psicologia).

"Acho que fazendo isso dá pra ver que tem muito mais coisas que tu não vai poder ver na universidade, mas ver isso na prática é diferente" (Hélio, formando, Psicologia).

"Eu acho que foi um dos fatores que me fez continuar aqui na Psicologia, porque eu estava meio desmotivada com as aulas. O laboratório, eu adorava a dinâmica, era todo mundo motivado e a gente aprendia várias coisas novas, entrava em contato com outras realidades, apresentar trabalho, viajar pra congressos, eu aprendi a fazer pesquisa, tive que fazer entrevista, transcrever, analisar. O laboratório veio pra mostrar uma ou- tra cara da Psicologia, foi o que me fez ficar, não quer dizer que o que eu vejo na faculdade é só o que existe" (Helena, formanda, Psicologia).

"Também, mesmo que tenha algum semestre que o aluno não está gostando das cadeiras, ele consiga permanecer, isso [participar de atividades extracurriculares] poderia ajudar a continuar..." (Hélio, formando, Psicologia).

Os relatos dos participantes deste estudo vão ao encontro dos resultados de outras pesquisas sobre atividades não obrigatórias, que indicam que a maioria dos estudantes tende a valorizar a busca por conhecimentos (Teixeira et al., 2008; Thiry et al., 2011). As vantagens apontadas pelos alunos entrevistados com o envolvimento com atividades extraclasse, como a possibilidade de aprofundar os conhecimentos dos conteúdos que consideram interessantes, de explorar as áreas de atuação da profissão e de desenvolver habilidades, também já foram encontradas por outros pesquisadores (Clark, Marsden, Whyatt, Thompson, \& Walker, 2015; Huang, \& Chang, 2004; Reaves et al., 2010; Thiry et al., 2011).

Nem todas atividades extracurriculares contribuem para o desenvolvimento de habilidades de forma equivalente (Clark et al., 2015) e para empregabilidade (Lau, Hsu, Acosta, \& Hsu, 2014). A participação em projetos de pesquisa, em particular, pode contribuir para o aperfeiçoamento da comunicação oral e escrita, do pensamento crítico e da resolução de problemas, além de favorecer o aprendizado das etapas da realização de uma pesquisa, da obtenção de financiamentos, da redação e da apresentação de trabalhos científicos (Thiry et al., 2011).

Ter um emprego remunerado parece aumentar conhecimentos sobre negócios, participar de grupos sociais desenvolve habilidades sociais em níveis mais elevados (Clarck et al., 2015). Outras experiências complementares podem auxiliar o estudante a adotar comportamentos e atitudes de um profissional, por meio da comunicação, independência no que se refere à realização do trabalho e à resolução de problemas, bem como da observação de profissionais coordenando projetos, orçamentos, prazos e equipes (Lau et al., 2014; Thiry et al., 2011).

De maneira geral, a participação dos estudantes em atividades extracurriculares, como a realização de estágios e no envolvimento em projetos de pesquisas 
aumenta o currículo, a rede de contatos e a autoeficácia profissional (Clark et al., 2015; Thiry et al., 2011), além de contribuir para a conquista do primeiro emprego e para a realização das atividades profissionais (Clark et al., 2015). Por um lado, os estudantes tendem a refinar e confirmar seus interesses e objetivos de carreira após avaliar as opções de atuação na profissão (Bardagi, \& Boff, 2010; Teixeira et al., 2007; Thiry et al., 2011). Por outro lado, os alunos podem mudar seus interesses, principalmente quando não recebem supervisão apropriada na realização dessas tarefas por orientadores/supervisores indisponíveis (Thiry et al., 2011). Assim, as atividades extracurriculares desempenham papel fundamental na permanência ou mudança do jovem na área de interesse e, inclusive, na profissão (Thiry et al., 2011).

Não é raro que os empregadores considerem os estudantes universitários preparados teoricamente, mas limitados na aplicação prática dos conhecimentos (Pascarella, \& Terenzini, 1991), pois esperam que os jovens apresentem capacidades adicionais junto à formação superior (Stevenson, \& Clegg, 2011), tais como habilidades interpessoais e estabelecimento de prioridades (Pascarella, \& Terenzini, 1991). O envolvimento em atividades extracurriculares é uma possibilidade de os estudantes desenvolverem as habilidades exigidas pelo mercado de trabalho, aumentando a probabilidade de ser empregado logo após a formatura (Clark et al., 2015; Pascarella, \& Terenzini, 1991; Stevenson, \& Clegg, 2011). Muitas universidades oferecem atividades não obrigatórias como oportunidades para os alunos desenvolverem habilidades de comunicação, de trabalho em equipe, de resolução de problemas e de liderança (Reaves et al., 2010). Então, cabe aos alunos escolher as oportunidades relacionadas aos conhecimentos, habilidades e experiências necessárias para ser um profissional com mais chances de sucesso no mercado de trabalho.

Alguns participantes identificaram dificuldades na busca por atividades extracurriculares. Dentre as dificuldades encontradas, os alunos destacaram não saberem onde procurar informações adicionais sobre os conteúdos trabalhados em aula (não sabiam quais livros poderiam ler) e não conseguir compreender os conteúdos estudados de forma independente, sem a explicação do professor, como limitações para o aprendizado autodidata. Obstáculos relacionados à gestão do tempo para conciliar estudos e outras atividades, bem como a oferta de poucas oportunidades pelo curso de graduação e o processo deficitário de divulgação de informações sobre essas oportunidades foram apontados pelos estudantes como limitadores no engajamento em atividades extracurriculares.

"O tempo é bem difícil, porque as aulas, às vezes eu não tenho aula de tarde, tenho só de manhã, então o tempo é um pouco quebrado" (Bianca, caloura, Psicologia).

"Só que pra mim isso foi bastante dificultoso, porque eu preciso de alguém que me ensine, então quando eu tinha que ir por conta se tornava mais difícil" (Carolina, formanda, Economia).

"Existem questões, como técnicas de pesquisa, que é muito fraco na Economia. A pessoa chega na monografia e não sabe o que fazer. A gente tem uma matéria, metodologia científica no primeiro semestre, e a gente via só filosofia em vez de ver a prática. [...] E o curso não oferece iniciação científica e depois tu é obrigada a fazer uma monografia sendo que tu nunca fizeste uma pesquisa" (Carolina, formanda, Economia).

"Aqui na Psicologia eu acho que é bem complicado, porque muita gente chega no final e nem sabia que existia. Se a gente não se fala entre a gente, a gente não saberia o que cada professor fazia, não teria noção disso. Então são espaços importantes e que são restritos a alguns alunos" (Letícia, formanda, Psicologia).

"Eu via outras pessoas fazendo isso [participando de projetos] e não tinha a oportunidade de fazer. Poderia ter procurado algum professor sim, só que eu noto que naquela época não tinha muita abertura por parte dos professores, principalmente se tu não tinhas uma posição definida em relação ao que tu gostava e se tu não tinha um conhecimento em relação àquela área que ele tava trabalhando" (Angélica, formanda, Psicologia).

Dificuldades encontradas pelos estudantes quanto à participação em atividades extracurriculares também foram encontradas em outros estudos (Teixeira et al., 2008; Thiry et al., 2011). Os obstáculos identificados pela literatura estão relacionados à necessidade de assumir uma atitude mais ativa na 
construção do conhecimento (Teixeira et al., 2008) e aos benefícios que os estudantes esperavam adquirir com o envolvimento em uma oportunidade, que não se realizou por meio da participação na mesma pela ausência de orientações dos supervisores (Thiry et al., 2011). Pode-se pensar que as pesquisas de Teixeira et al. (2008) e de Thiry et al. (2011), ambas com delineamento qualitativo e com estudantes universitários, apontam o desamparo sentido pelos alunos para iniciar a busca por conhecimentos de forma independente como a fonte das dificuldades. O mesmo sentimento de desamparo dos alunos (por parte dos professores e da instituição) foi verificado na presente pesquisa, quando os jovens afirmam não saber onde buscar oportunidades e conhecimentos e constatam que a instituição oferecia poucas opções. A percepção dos estudantes sobre a falta de instrução e apoio por parte dos professores e da instituição pode prejudicar a adaptação dos mesmos ao ensino superior, visto que esses vínculos são aspectos constituintes da adaptação acadêmica.

Os estudantes ingressam na universidade em busca de uma preparação profissional de qualidade, tanto no que se refere à titulação quanto ao crescimento e realização pessoal (Pachane, 2003). Dessa forma, experiências deficitárias podem levar os alunos a perder o interesse e a abandonar seus objetivos de carreira (Thiry et al., 2011). A partir disso, recomenda-se que as universidades ofereçam e/ou divulguem oportunidades de participar de experiências que promovam a responsabilidade frente ao trabalho supervisionada por profissionais, além de estimularem o contato com pessoas, conteúdos e ambientes relevantes à área de formação e à carreira (Bardagi, \& Hutz, 2010; Huang, \& Chang, 2004; Thiry et al., 2011).

\section{Considerações finais}

O objetivo deste estudo foi identificar o que induz os estudantes universitários a buscarem a realização de atividades extracurriculares, em que tipo de atividades se envolvem e como avaliam esse envolvimento. Verificou-se que os motivos para o envolvimento dos participantes em atividades extracurriculares são a necessidade de buscar conhecimentos para complementar sua formação, a percepção da falta de domínio de conteúdos e da necessidade de entrar em contato com a profissão. Além de buscar conhecimento em artigos e livros, os estudantes rela- taram participar de cursos de informática, trabalho voluntário, projetos de pesquisa e extensão, eventos e estágios não obrigatórios. Observou-se ainda que os formandos parecem ter mais opções nesse sentido do que os calouros, provavelmente pelo fato dos primeiros estarem mais familiarizados com o ambiente universitário e com as oportunidades ligadas ao curso do que os últimos, ainda em fase de adaptação. Os participantes avaliaram o envolvimento em atividades extracurriculares como necessário e indicaram vantagens como autonomia, exploração de áreas de atuação na profissão, desenvolvimento de habilidades e preparação para o trabalho de conclusão de curso. Entretanto, os estudantes podem encontrar dificuldades para participar de atividades não obrigatórias, seja por limitações pessoais (como falhas na gestão do tempo ou em habilidades pessoais para conseguir se engajar nas atividades) ou por questões institucionais (como a escassez ou falha na divulgação das oportunidades).

Sugere-se cautela na interpretação destes resultados, uma vez que a pesquisa possui algumas limitações. A amostra foi composta predominantemente por mulheres de apenas dois cursos de graduação de uma universidade pública. Assim, sugere-se que mais pesquisas sejam desenvolvidas comparando as percepções de estudantes provenientes de áreas e de universidades diferentes sobre as atividades extracurriculares realizadas durante a trajetória universitária e sobre como essas podem ter influenciado na adaptação acadêmica dos mesmos. Acredita-se que pode haver diferenças na percepção de estudantes de universidades públicas e privadas no que se refere à divulgação das oportunidades aos estudantes de forma igualitária.

Já se sabe que a exploração profissional contribui para o planejamento de carreira dos estudantes universitários, pois a posse de informações possibilita uma decisão mais realista. No entanto, a percepção dos estudantes sobre o processo da busca por atividades extracurriculares (motivo da busca, tipo de atividades e avaliação da participação) não havia sido investigado de forma qualitativa no Brasil. Também não ficavam claras as semelhanças e diferenças nas percepções de calouros e formandos sobre sua participação em atividades extracurriculares e sobre os obstáculos encontrados para o envolvimento com as mesmas. Assim, os resultados deste estudo podem ampliar o conhecimento sobre o papel da exploração profissional na retenção e 
no envolvimento de estudantes com o curso, bem como no desenvolvimento da habilidade de buscar aprendizagem de forma mais independente, especialmente para o período pós-formatura, no qual os estudantes se preocupam com a inserção no mercado de trabalho e com a atualização dos seus conhecimentos profissionais.

Os conhecimentos produzidos por este estudo ainda podem ser úteis para psicólogos no exercício de sua profissão. Os psicólogos que trabalham em Instituições de Ensino Superior, em órgãos responsáveis pelo apoio e/ou atendimento ao estudante, podem considerar essas informações para propor intervenções voltadas à recepção de calouros com foco na apresentação e adaptação à universidade. A apresentação das oportunidades e das possibilidades de atividades extracurriculares durante o período universitário podem contribuir para maior adaptação dos alunos, uma vez que podem favorecer um maior envolvimento com o curso superior e com planejamento da trajetória acadêmica. A promoção de feiras de oportunidades que apresentem atividades extracurriculares dentro da universidade (participação em projetos de pesquisa e extensão, bolsas) e fora dela (cursos, estágios, trabalhos voluntários, eventos)

\section{Referências}

Alves, A. F., Gonçalves, P., \& Almeida, L. S. (2012). Acesso e sucesso no ensino superior: inventariando as expectativas dos estudantes. Revista Galego-Portuguesa de Psicoloxía e Educación, 20(1), 121-131. Recuperado de http://ruc.udc.es/dspace/bitstream/handle/2183/12112/RGP_20_2012_art_8.pdf?sequence=1

Baker, C. N. (2008). Under-represented college students and extracurricular involvement: the effects of various student organizations on academic performance. Social Psychology of Education, 11(3), 273-298. doi:10.1007/s11218-007-9050-y

Bardagi, M. P., \& Boff, R. M. (2010). Autoconceito, autoeficácia profissional e comportamento exploratório em universitários concluintes. Avaliação : Revista da Avaliação da Educação Superior (Campinas), 15(1), 41-56. doi:10.1590/S1414-40772010000100003

Bardagi, M. P., \& Hutz, C. S. (2010). Satisfação de vida, comprometimento com a carreira e exploração vocacional em estudantes universitários. Arquivos Brasileiros de Psicologia, 62(1), 159-170. Recuperado de http://pepsic.bvsalud.org/scielo.php?script=sci_arttext\&pid=S1809-52672010000100016 pode ampliar os conhecimentos dos estudantes sobre as áreas de atuação do profissional.

Ações voltadas para estudantes em final da graduação podem possibilitar a reflexão sobre as experiências vivenciadas até então. Essa reflexão permite a conversão das experiências extracurriculares em habilidades profissionais para a inserção no mercado de trabalho. Supõe-se que esse exercício pode contribuir não só para a elaboração dos currículos dos formandos, mas também para a autoeficácia profissional dos mesmos ao avaliar sua trajetória acadêmica.

Além disso, o conhecimento do impacto das atividades extracurriculares na formação dos estudantes pode auxiliar em atendimentos individuais para alunos insatisfeitos com o curso. Avaliar a iniciativa e envolvimento do estudante na busca por oportunidades extraclasse pode contribuir para uma decisão melhor informada sobre permanência ou evasão do curso de graduação. Em outras palavras, o envolvimento com atividades extracurriculares pode amparar a permanência de estudantes universitários na graduação cuja queixa seja o distanciamento entre os conteúdos trabalhados em aula e a profissão.

Bardagi, M. P., \& Hutz, C. S. (2012). Rotina acadêmica e relação com colegas e professores: Impacto na evasão universitária. Psico, 43(2), 174-184. Recuperado de http://revistaseletronicas.pucrs.br/fo/ojs/index. $\mathrm{php} /$ revistapsico/article/view/7870

Bardin, L. (2011). Análise de conteúdo. Lisboa: Edições 70. Bridi, J. C. A. (2010). Atividade de pesquisa: contribuições da iniciação científica na formação geral do estudante universitário. Olhar de Professor, 13(2), 349-360. doi:10.5212/OlharProfr.v.13i2.0010

Busseri, M. A., Rose-Krasnor, S., Pratt, M. W., Adams, G. R., Birnie-Lefcovitch, S., Polivy, J. et al. (2010). A longitudinal study of breadth and intensity of activity involvement and the transition to university. Journal of Research on Adolescence, 21(2), 512-518. doi:10.1111/j.1532-7795.2010.00691.x

Clark, G., Marsden, R., Whyatt, J. D., Thompson, L., \&Walker, M. (2015). "It's everything else you do...": Alumni views on extracurricular activities and employability. Active Learning in Higher Education, 16(2), 133-147. doi:10.1177/1469787415574050

Credé, M., \& Niehorster, S. (2012). Adjustment to college as measured by the student adaptation to college questionnaire: a quantitative review of its 
structure and relationships with correlates and consequences. Educational Psychology Review, 24(1), 133-165. doi:10.1007/s10648-011-9184-5

Elias, K., \& Drea, C. (2013). The co-curricular record: enhancing a post-secondary education. College Quarterly, 16(1). Recuperado de http://collegequarterly.ca/2013-vol16-num01-winter/elias-drea.html

Fior,C.A.,\&Mercuri,E. (2009). Formaçãouniversitária eflexibilidade curricular: Importância das atividades obrigatórias e não obrigatórias. Psicologia da Educação, 29, 191215. Recuperado de http://pepsic.bvsalud.org/scielo. php?script=sci_arttext\&pid=S1414-69752009000200010

Hu, S., \& Wolniak, G. C. (2010). Initial evidence on the influence of college student engagement on early career earnings. Research in Higher Education, 51(8), 750-766. doi:10.1007/s11162-010-9176-1

Huang, Y. R., \& Chang, S. M. (2004). Academic and cocurricular involvement: Their relationship and the best combinations for student growth. Journal of College Student Development, 45(4), 391-406. doi:10.1353/csd.2004.0049

Igue, E. A., Bariani, I. C. D., \& Milanesi, P. V. B. (2008). Vivência acadêmica e expectativas de universitários ingressantes e concluintes. Psico-USF, 13(2), 155-164. doi:10.1590/S1413-82712008000200003

Jordaan, J. P. (1963). Exploratory behavior: The formation of self and occupational concepts. In D. Super, R. Starishevsky, N. Matlin, \& J. P. Jordaan (Eds.), Career development: selfconcept theory: essays in vocational development (pp. 42-78). NewYork: College Entrance Examination Board.

Lau, H.-H., Hsu, H.-Y., Acosta, S., \& Hsu, T.-L. (2014). Impact of participation in extra-curricular activities during college on graduate employability: an empirical study of graduates of Taiwanese business schools. Educational Studies, 40(1), 26-47. doi:10.1080/03055698.2013.830244

Minayo, M. C. S. (1992). Desafio do conhecimento: pesquisa qualitativa em saúde. São Paulo, SP: Hucitec.

Ogushi, M. M. P., \& Bardagi, M. P. (2015). Reflexões sobre a relação estudante-universidade a partir de uma experiência de atendimento em orientação profissional. Extensio, 12(19), 33-50. doi:10.5007/1807-0221.2015v12n19p33

Oliveira, M. C., \& Coleta, M. F. D. (2008). Adaptação e validação da Escala Combinada de Atitudes da Maturidade de Carreira (CDA): versão para estudantes universitários. Revista Brasileira de Orientação Profissional, 9(2), 45-65. Recuperado de http:// pepsic.bvsalud.org/scielo.php?script=sci_arttext\&pid=S1679-33902008000200006

Pachane, G. G. (2003). A experiência universitária e sua contribuição ao desenvolvimento pessoal do aluno. In E. Mercuri, \& S. A. J. Polydoro (Orgs.), Estudante universitário: características e experiências de formação (pp. 155-186). Taubaté, SP: Cabral.

Pascarella, E. T., \& Terenzini, P. T. (1991). How college affects students: a third decade of research. San Francisco: Jossey-Bass.

Peres, C. M., Andrade, A. S., \& Garcia, S. B. (2007). Atividades extracurriculares: multiplicidade e diferenciação necessárias ao currículo. Revista BrasileiradeEducaçãoMédica, 31(3), 147-155. doi:10.1590/S0100-55022007000300002

Piovesan, A., \& Temporini, E. R (1995). Pesquisa exploratória: procedimento metodológico para o estudo de fatores humanos no campo da saúde pública. Revista de Saúde Pública, 29(4), 318-325. doi:10.1590/S0034-89101995000400010

Rawat, N., Rastogi, A., Jaiswal, K., \& Nigam, A. (2014, November). Analysis of relationship between extracurricular activities and academic performance by computational intelligence. In Conference: 2014 Innovative Applications of Computational Intelligence on Power, Energy and Controls with their impact on Humanity (CIPECH); 28-29 nov 2014. Recuperado de http://ieeexplore.ieee.org/xpl/articleDetails.jsp?arnumber $=7019087$

Reaves, D. W., Hinson, R. A., \& Marchant, M. A. (2010). Benefits and costs of faculty participation in extraand co-curricular activities. NACTA Journal, 54(1), 54-60. Recuperado de http://connection.ebscohost. $\mathrm{com} / \mathrm{c} /$ articles $/ 48916947$ / benefits-costs-faculty-participation-extra-co-curricular-activities

Santos, A. A. A., Mognon, J. F., Lima, T. H., \& Cunha, N. B. (2011). A relação entre vida acadêmica e a motivação para aprender em universitários. Revista Semestral da Associação Brasileira de Psicologia Escolar e Educacional, 15(2), 283-290. Recuperado de http://www.scielo.br/pdf/pee/v15n2/v15n2a10

Soares, A. B., Francischetto, V., Dutra, B. M., Miranda, J. M., Nogueira, C. C., Leme, V. et al. (2014). O impacto das expectativas na adaptação acadêmica dos estudantes no Ensino Superior. Psico-USF, 19(1), 49-60. doi:10.1590/S1413-82712014000100006

Soares, A. B., Poube, L. N., \& Mello, T. V. S. (2009). Habilidades sociais e adaptação acadêmica: um estudo comparativo em instituições de ensino público e 
privado. Aletheia, 29, 27-42. Recuperado de http:// pepsic.bvsalud.org/scielo.php?script=sci_arttext\&pi$\mathrm{d}=$ S1413-03942009000100004

Soares, A. P. C., Almeida, L. S., \& Ferreira, J. A. (2002). Contributos para a validação do Inventário de Desenvolvimento da Autonomia de Iowa com estudantes universitários portugueses. Revista Psicologia e Educação, 1(1/2), 91-106. Recuperado de http:// repositorium.sdum.uminho.pt/handle/1822/12099

Sparta, M., Bardagi, M. P., \&Andrade,A.M.J. (2005).Exploração vocacional e informação profissional percebida em estudantes carentes. Aletheia, (22), 79-88. Recuperado de http://pepsic.bvsalud.org/scielo.php?scrip$\mathrm{t}=$ sci_arttext\&pid=S1413-03942005000200008

Stevenson, J., \& Clegg, S. (2011). Possible selves: students orientating themselves towards the future through extracurricular activity. British Educational Research Journal, 37(2), 231-246. doi:10.1080/01411920903540672

Tavares, D. M. (2012). Adaptação ao ensino superior e otimismo em estudantes do $1^{\circ}$ ano (Dissertação de mestrado). Universidade Fernando Pessoa, Porto, 2012.

Teixeira, M. A. P., \& Gomes, W. B. (2005). Decisão de carreira entre estudantes em fim de curso universitário. Psicologia: Teoria e Pesquisa, 21(3), 327-334. doi:10.1590/S0102-37722005000300009

Teixeira, M. A. P., Bardagi, M. P., \& Hutz, C. S. (2007). Escalas de exploração vocacional (EEV) para universitários. Psicologia em Estudo, 12(1), 195-202. doi:10.1590/S1413-73722007000100023

Teixeira, M. A. P., Dias, A. C. G., Wottrich, S. H., \& Oliveira, A. M. (2008). Adaptação à universidade em jovens calouros. Revista Semestral da Associação Brasileira de Psicologia Escolar e Educacional, 12(1), 185-202. doi:10.1590/S1413-85572008000100013

Thiry, H., Laursen, S. L., \& Hunter, A. B. (2011). What experiences help students become scientists?: a comparative study of research and other sources of personal and professional gains for STEM undergraduates. The Journal of Higher Education, 82(4), 357-388. doi:10.1353/jhe.2011.0023

\section{Clarissa Tochetto de Oliveira}

Doutoranda pela Universidade Federal do Rio Grande do Sul, Porto Alegre - RS. Brasil.

E-mail: clarissa.tochetto@gmail.com

\section{Anelise Schaurich dos Santos}

Doutoranda pela Unisinos, São Leopoldo - RS. Brasil. E-mail: anelise_ssantos@hotmail.com

\section{Ana Cristina Garcia Dias}

Doutora em Psicologia pela Universidade de São Paulo, São Paulo - SP. Docente da Universidade Federal de Santa Maria, Santa Maria - RS. Brasil.

E-mail: anacristinagarciadias@gmail.com

Endereço para envio de correspondência: Rua Ramiro Barcelos, 2600. Bairro Rio Branco. CEP 90035003 - Porto Alegre, RS - Brasil.

Recebido 10/01/2015

Reformulação 15/08/2016

Aprovado 04/11/2016

Received $01 / 10 / 2015$

Reformulated $08 / 15 / 2016$

Approved 11/04/2016

Recibido 10/01/2015

Reformulado 15/08/2016

Aceptado 04/11/2016

Como citar: Oliveira, C. T., \& Santos, A. S. (2016). Percepções de estudantes universitários sobre a realização de atividadesextracurricularesnagraduação. Psicologia:CiênciaeProfissão,36(4):864-876.doi:10.1590/1982-3703003052015

How to cite: Oliveira, C. T., \& Santos, A. S. (2016). College students' perceptions regarding involvement in extra-curricular activities during college. Psicologia: Ciência e Profissão, 36(4): 864-876. doi:10.1590/1982-3703003052015

Cómo citar: Oliveira, C. T., \& Santos, A. S. (2016). Percepciones de estudiantes universitarios sobre participación en actividades extracurriculares en la pregrado. Psicologia: Ciência e Profissão, 36(4): 864-876. doi:10.1590/1982-3703003052015 